\title{
Recent experiments conducted with the Wide-field Imaging Interferometry Testbed (WIIT)
}

David T. Leisawitz ${ }^{\mathrm{a}}$, Roser Juanola-Parramon ${ }^{\mathrm{a}, \mathrm{b}}$, Matthew Bolcar ${ }^{\mathrm{a}}$, James R. Fienup ${ }^{\mathrm{c}}$, Alexander S. Iacchetta ${ }^{\mathrm{c}, \mathrm{d}}$, Stephen F. Maher, ${ }^{\mathrm{a}, \mathrm{e}}$, and Stephen A. Rinehart ${ }^{\mathrm{a}}$

${ }^{a}$ NASA Goddard Space Flight Center, 8800 Greenbelt Rd., Greenbelt, MD, USA 20771-2400

${ }^{b}$ NASA Postdoctoral Program Fellow

${ }^{\mathrm{c}}$ Institute of Optics, Univ. of Rochester, 275 Hutchison Rd., Rochester, NY, USA 14627-0186

${ }^{\mathrm{d}}$ NASA Space Technology Research Fellow

${ }^{\mathrm{e}} \mathrm{SSAI}$, Inc.

\begin{abstract}
The Wide-field Imaging Interferometry Testbed (WIIT) was developed at NASA's Goddard Space Flight Center to demonstrate and explore the practical limitations inherent in wide field-of-view "double Fourier" (spatio-spectral) interferometry. The testbed delivers high-quality interferometric data and is capable of observing spatially and spectrally complex hyperspectral test scenes. Although WIIT operates at visible wavelengths, by design the data are representative of those from a space-based far-infrared observatory. We used WIIT to observe a calibrated, independently characterized test scene of modest spatial and spectral complexity, and an astronomically realistic test scene of much greater spatial and spectral complexity. This paper describes the experimental setup, summarizes the performance of the testbed, and presents representative data.
\end{abstract}

Keywords: Interferometry, WIIT, Spatio-spectral interferometry, Double Fourier interferometry

\section{EXPERIMENTAL SETUP}

WIIT is a visible wavelength double Fourier interferometer located in the Advanced Interferometry and Metrology Lab (AIM) at NASA's Goddard Space Flight Center. ${ }^{1}$ Data obtained with WIIT in the AIM Lab are practically free of environmentally-induced errors (wavefront distortion due to air turbulence, temperature and humidity fluctuations, and vibrations). The data quality is limited by photon noise from the test scene and wellunderstood instrumental error terms. WIIT provides nearly full $u-v$ plane coverage and a wide field of view. The testbed data are highly representative of data one would expect to obtain with a space-based far-infrared spatiospectral interferometer.

Figure 1 shows the path followed by light through the testbed. Light from a test scene located at the focus of a 21 inch diameter collimating mirror is projected into the interferometer, emulating a source in the sky. Two elliptical flat collector mirrors $(25 \mathrm{~mm}$ in projection, as seen from the collimating mirror) intercept separate portions of the incident wavefront, establishing an interferometric baseline. The distance between the collector mirrors is adjusted to vary the baseline length, and the test scene is rotated to vary the baseline orientation, enabling a full exploration of the $u$-v plane. The maximum baseline length is about $230 \mathrm{~mm}$, so the achievable angular resolution (ability to

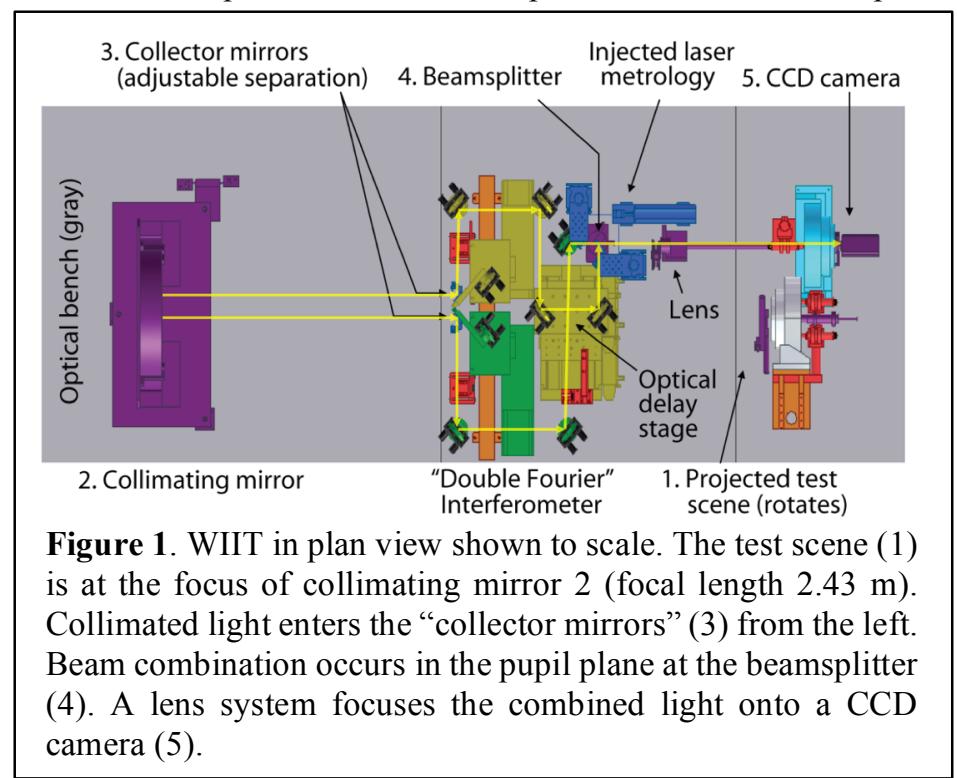


distinguish adjacent point sources) is $\theta_{\text {res }}=\lambda / 2 b_{\max }=0.27 \operatorname{arcsec}$ at $\lambda=600 \mathrm{~nm}$. Two 25 -mm diameter $(D)$ collimated beams traverse the WIIT interferometer, starting at the collector mirrors and reflecting off flat mirrors until they merge orthogonally and combine at a half-reflective/half-transmissive parallel plate beamsplitter. The primary beam width $\theta_{p}(=1.2 \lambda / D)$ is 6 arcsec. Each WIIT CCD camera pixel subtends 1.6 arcsec. The WIIT optical delay line has a long scan range that can provide spectral resolution $>3000$ across the FOV.

The Calibrated Hyperspectral Image Projector (CHIP) $)^{2}$ provides a hyperspectral scene to WIIT with spatial features as small as 0.16 arcsec (i.e., unresolved even at the resolution limit of the interferometer), spectral features as narrow as $5 \mathrm{~nm}(\lambda / \Delta \lambda \sim 120)$, and customized spectra pixel by pixel over the wavelength range 380 to $780 \mathrm{~nm}$. The CHIP is installed in WIIT at the location of the test scene (1) in Fig. 1. The effective dynamic range of WIIT with CHIP is $\sim 10^{5}$, enabling observations of astronomically interesting scenes, such as dusty debris disks. In such disks, a typical star-to-disk contrast ratio is $\sim 10^{4}$ in the far-IR.

A computational optical system model of WIIT enables us to understand the contributions of individual instrumental error terms. Imperfect mirrors and alignment are the main contributors to a predicted net $18 \%$ loss of fringe visibility relative to a perfect interferometer. We confirmed experimentally that, when an unresolved source (nominal visibility, $V=1$ ) is observed with WIIT, the actual measured visibility is 0.82 , as expected. Photon shot noise from the observed scene is the dominant contributor to visibility uncertainty. We have used the computational optical system model to produce synthetic interferograms corresponding to observations of simple sources, and the synthetic interferograms closely match real WIIT measurements. Independent measurements of the spatial and spectral content of the test scene serve as a measure of "truth," and eventually can be compared directly with reconstructed hyperspectral data cubes based on measured and synthetic interferometric data.

\section{THE TEST SCENES}

\subsection{Scene 1: A scene of modest spatial and spectral complexity}

We used WIIT and CHIP to observe the test scene described in Figures 2 and 3. As illustrated schematically in Figure 2, the scene contains six pairs of circular sources, each of which can be pictured as a "binary star." The individual "stars" all have the same diameter, 6 CHIP pixels $(0.98$ arcsec). The center-to-center separations between the stars vary from 2 CHIP pixels (largely overlapping stars) to 12 CHIP pixels (i.e., edge-to-edge separation of one "stellar" diameter). The six binary stars are spaced at intervals of 77 CHIP pixels ( $\sim 13$ arcsec $)$. Given the primary beam width, $\theta_{p}$, and the interferometric resolution, $\theta_{\text {res }}$, mentioned above, the binary stars - even the pairs with the widest separation - are point sources to the individual apertures, but the individual stars are interferometrically resolvable. Each binary star is separated from its neighboring star pair by $\sim 2 \theta_{p}$, so the scene covers a wide field of view on the WIIT CCD camera.

Five different spectra, labeled A through E, are projected with CHIP. The spectrum varies from star to star, as shown in Fig. 2.

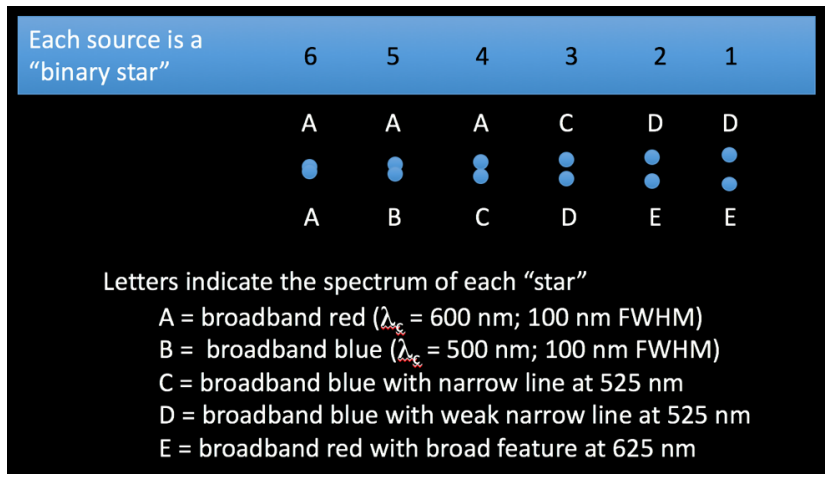

Figure 2. Above: spatial layout of sources in test scene 1 (not to scale). Right: Nominal spectra used in the test scene at locations indicated above.

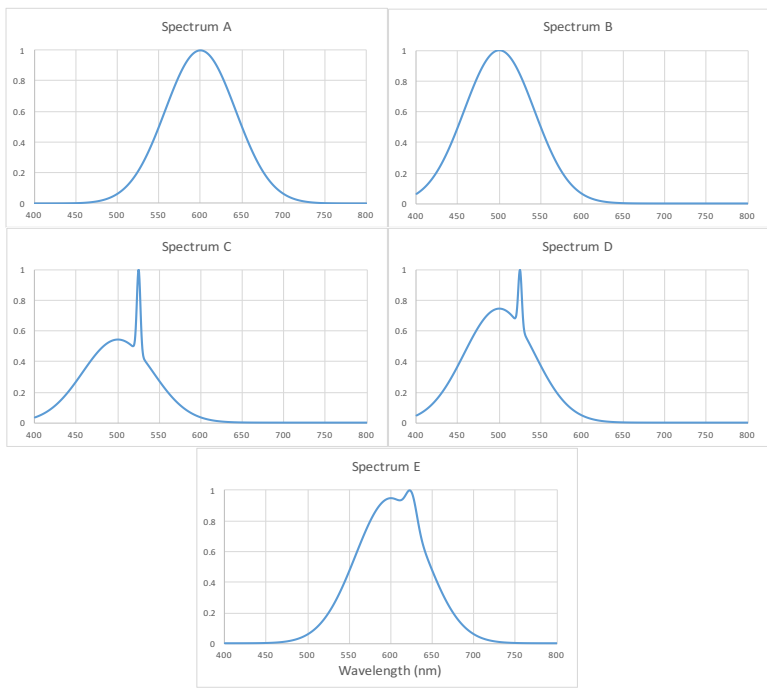


Figure 3 shows the test scene as viewed at low resolution $\left(\theta_{p}\right)$ on the WIIT CCD camera. Four phase reference sources, arranged in a square pattern, surround the central part of the field. The phase reference sources each measure one CHIP pixel in diameter. Between each pair of phase reference sources, a miniature "binary star" comprising two illuminated CHIP pixels separated by a single dark pixel. All of these small sources have the same spectrum, and the spectrum is a blend of spectra $\mathrm{A}-\mathrm{E}$.

Direct spectroscopic measurements of the CHIP output provide a measure of "truth" and indicate that the actual projected spectra are very close approximations to the nominal spectra shown in Fig. 2. Likewise, direct high-resolution images of the CHIP output (not yet implemented) will provide ground truth for the spatial content of the test scene.

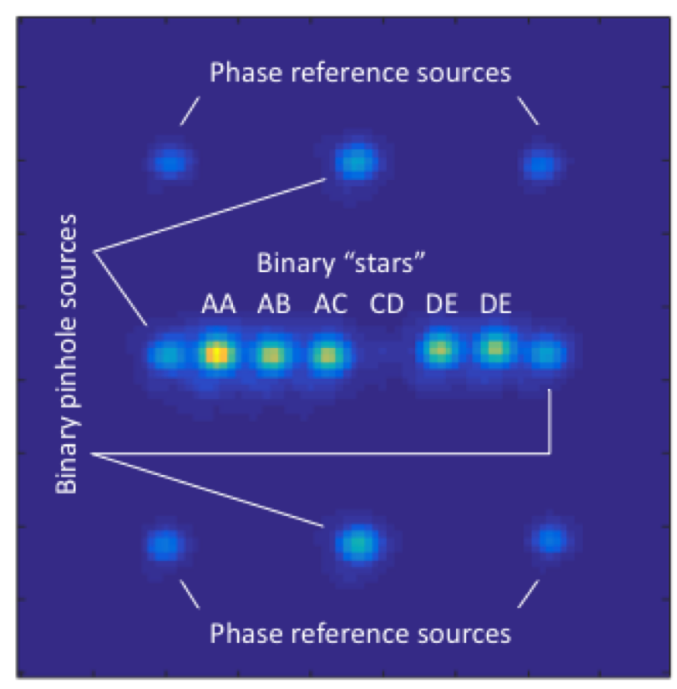

Figure 3. Test scene 1 as it appears on the WIIT CCD camera, labeled to show locations of the binary "stars," binary pinhole sources, and phase reference sources. Source "CD" is very faint.

\subsection{Scene 2: A simulated far-infrared extragalactic deep field}

We also used WIIT and CHIP to observe an astronomically realistic test scene based on a simulated far-infrared deep field observation of the extragalactic sky (Figure 4). The original simulation is a hyperspectral cube covering a 1.2 arcmin patch of sky in which the spatial pixels each subtend 0.07 arcsec and the spectral channels span the wavelength range 25 to $400 \mu \mathrm{m}$ in $1 \mu \mathrm{m}$ intervals. The simulated sky contains spatially uniform zodiacal emission calibrated to the COBE DIRBE zodiacal emission model; Galactic cirrus emission was normalized to IRAS observations at $>1$ arcmin angular scales, and extrapolated to finer scales using a power law distribution; and galaxies were distributed in redshift and luminosity according to distribution functions calibrated before the

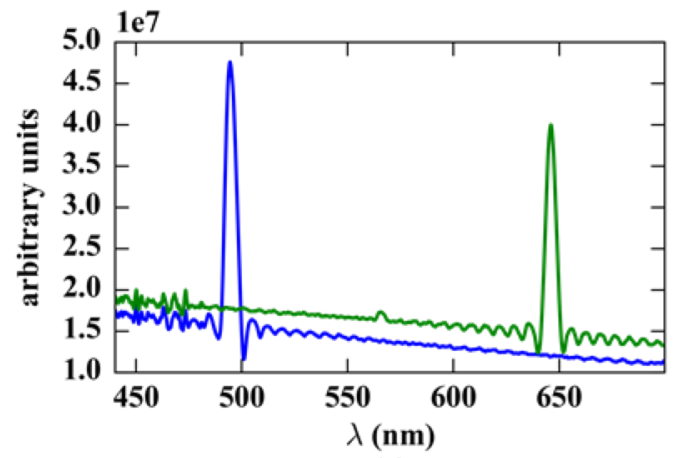

(a)

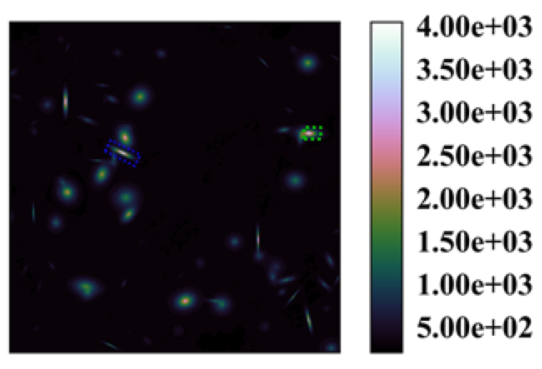

(b)

Figure 4. To prepare test scene 2, we began with a simulated hyperspectral far-IR extragalactic deep field, broke the data cube into four wavelength bins, mapped the far-IR wavelengths into the visible range for projection with CHIP, and used 8 eigenspectra and corresponding eigenimages to capture most of the original spatial and spectral content while enabling time-efficient projection with the CHIP. (a) CHIP projected spectra (blend of eigenspectra) in the pixels outlined in matching colors in image (b), showing the presence of continuum (zodiacal, cirrus, and galactic) and spectral line emission (from the galaxies at their respective redshifts) from the original far-IR spectral range $307-400 \mu \mathrm{m}$. (b) A wavelength-integrated image representing the projected $280 \mathrm{x}$ 280-pixel test scene. Four separate WIIT data sets were collected, one for each of the four wavelength bins, but only one of the four is represented here. 
Herschel mission. Each galaxy has a standard morphology and spectral template that includes both continuum and spectral lines.

To project this far-infrared scene for observation with WIIT, the original hyperspectral data cube was regridded to the CHIP resolution, a $280 \times 280$ CHIP pixel sub-field was selected, and the simulated far-IR wavelengths were mapped into the visible wavelength range accessible to CHIP. Apart from this transformation, we aimed for a highfidelity representation of the scene. We found that nearly all of the spatial and spectral information could be preserved if the field was divided into four wavelength bins, and 8 eigenspectra and corresponding eigenimages were used to enable time-efficient projection with the CHIP. ${ }^{3}$ Figure 4 illustrates the richness of the hyperspectral scene corresponding to the original $307-400 \mu \mathrm{m}$ wavelength bin.

\section{THE EXPERIMENTS}

Table 1 summarizes the WIIT observational setup applied to five experiments, one for scene 1 , and four corresponding to the far-IR wavelength bins that collectively represent test scene 2 . In each case, the $u$ - $v$ plane was densely sampled.

Table 1. Experimental setup used to observe each scene

\begin{tabular}{|c|c|c|c|c|c|}
\hline Scene & $\begin{array}{l}\text { Number of } \\
\text { baselines } \\
\text { observed }\end{array}$ & $\begin{array}{l}\text { CCD } \\
\text { pixels }\end{array}$ & $\begin{array}{l}\text { FoV } \\
\text { (arcmin) }\end{array}$ & $\begin{array}{l}\text { Optical delay } \\
\text { sampling } \\
\text { interval }(\mathrm{nm})\end{array}$ & $\begin{array}{l}\text { Optical delay } \\
\text { range }(\mu \mathrm{m})\end{array}$ \\
\hline 1 & 420 & $90 \times 90$ & 2.4 & 100 & 240 \\
\hline $2(25-118 \mu \mathrm{m})$ & 421 & $90 \times 90$ & $\begin{array}{r}2.4 \text { total, } \\
0.75 \text { science }\end{array}$ & 72 & 280 \\
\hline $2(119-212 \mu \mathrm{m})$ & 421 & $90 \times 90$ & $\begin{array}{r}2.4 \text { total, } \\
0.75 \text { science }\end{array}$ & 87 & 240 \\
\hline $2(213-306 \mu \mathrm{m})$ & 421 & $90 \times 90$ & $\begin{array}{r}2.4 \text { total, } \\
0.75 \text { science }\end{array}$ & 83 & 240 \\
\hline $2(307-400 \mu \mathrm{m})$ & 421 & $90 \times 90$ & $\begin{array}{r}2.4 \text { total, } \\
0.75 \text { science }\end{array}$ & 124 & 240 \\
\hline
\end{tabular}

Scene 1 was measured at 420 interferometric baselines. Interferometric data corresponding to each sampled baseline were captured in a single FITS file. Baselines ranging from $36 \mathrm{~mm}$ to $226 \mathrm{~mm}$ in $10 \mathrm{~mm}$ increments (less than half of the collector mirror diameter) were observed, and multiple baseline position angles were sampled at each baseline length. At $226 \mathrm{~mm}$, for example, position angles ranging from 0 to 179.9 degrees were measured at increments of 5.1 degrees, for a total of 36 baseline samples. Fewer position angles were sampled at shorter baseline lengths. At each baseline, the optical delay line was scanned while WIIT CCD camera frames from a $90 \mathrm{x} 90$-pixel area (corresponding to a square FoV 2.4 arcmin on a side) were captured at a frequency designed to sample the optical delay in increments of approximately $100 \mathrm{~nm}(<\lambda / 4)$. Each FITS file contains a data cube with two dimensions corresponding to the WIIT CCD pixels, and a third dimension corresponding to 2404 time samples covering a $240 \mu \mathrm{m}$ range in optical path difference. An injected laser metrology system provided real-time measurements of the true optical path difference (accurate to $10 \mathrm{~nm}$ ) at the time of each camera exposure during the delay line scan. Metrology and housekeeping data, as well as metadata and camera dark frames, are included in the FITS files.

Each of the four far-IR wavelength bins comprising test scene 2 was similarly observed, as summarized in Table 1. Again, camera frames from a 90 x 90 pixel area were captured, but the simulated far-IR deep field subtended only $28 \times 28$ pixels, or 0.75 arcmin out of the 2.4 arcmin overall FoV. Within this science field, accounting for the four wavelength bins, we captured 4 (wavelength bins) x 421 (baselines) x 28 x 28 (pixels), or 1.3 million interferograms. To facilitate experimentation with alternative approaches to hyperspectral reconstruction, phase reference and calibration sources were distributed in the wider field surrounding the science field.

To illustrate the data quality, two representative interferograms, corresponding to observations of scene 1 from a single CCD pixel in each of two FITS files, are shown in Figure 5. Both interferograms correspond to observations 
of binary star number 5 (spectrum "AB"; see Fig. 3) at baseline lengths $56 \mathrm{~mm}$ and $106 \mathrm{~mm}$, with the baseline oriented approximately parallel to the direction in which the stars are separated from each other. The binary pair is marginally resolved at $56 \mathrm{~mm}$ and better resolved at the longer baseline, accounting for the detected difference in fringe visibility and in the shapes of the two interferograms.

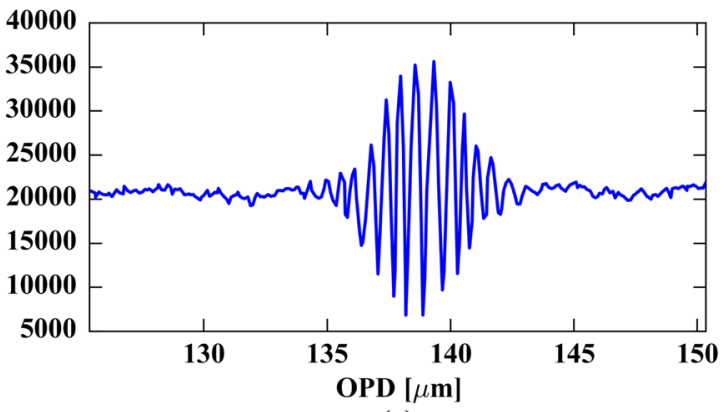

(a)

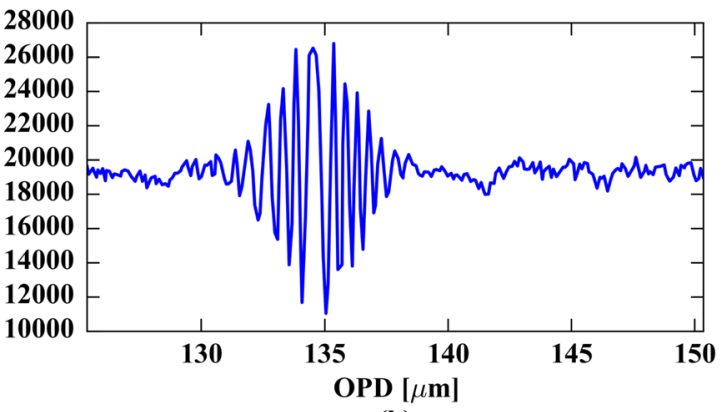

(b)

Figure 5. Two interferograms from a single WIIT CCD pixel for the "binary star" source "AB." (a) $56 \mathrm{~mm}$ interferometric baseline. (b) $106 \mathrm{~mm}$ baseline.

\section{SIMULATION AND HYPERSPECTRAL RECONSTRUCTION}

Dr. Juanola-Parramon adapted the FIInS double-Fourier interferometry simulator to model the WIIT observations of test scene $1 .^{4}$ The model successfully predicts interferograms corresponding to the data we collected in the lab, and we have demonstrated hyperspectral reconstruction based on direct Fourier transformation of the simulated data. ${ }^{4}$ We will soon attempt reconstruction based on data obtained in the lab.

To foster the development of spatio-spectral interferometry, data from the experiments described above will be provided on request.

\section{REFERENCES}

[1] Leisawitz, D., Bolcar, M., Lyon, R., Maher, S., Memarsadeghi, N., Rinehart, S., and Sinukoff, E. 2012, "Developing wide-field spatio-spectral interferometry for far-infrared space applications," in Optical and Infrared Interferometry III, eds. F. Delplancke, J.K. Rajagopal, \& F. Malbet, Proc. SPIE 8445, 84450A.

[2] Bolcar, M.R., Leisawitz, D.T., Maher, S.F., and Rinehart, S.A. 2012, "Demonstration of the wide-field imaging interferometer testbed using a calibrated hyperspectral image projector," in Optical and Infrared Interferometry III, eds. F. Delplancke, J.K. Rajagopal \& F. Malbet, Proc. SPIE, 8445, 84452D-84452D-9.

[3] Iacchetta, A.S., Fienup, J.R., Leisawitz, D.T., and Bolcar, M.R. 2015, "Nonnegative matrix factorization for efficient hyperspectral image projection," in Imaging Spectrometry XX, eds. T.S. Pagano \& J.F. Silny, Proc. SPIE, 9611, 96110Y.

[4] Juanola-Parramon, J., Leisawitz, D.T., Bolcar, M.R., Maher, S.F., Rinehart, S.A., Iacchetta, A., and Savini, G. 2016, in Optical and Infrared Interferometry and Imaging V, eds. F. Malbet, M.J. Creech-Eakman, \& P.G. Tuthill, Proc. SPIE 9907, this volume. 